\title{
Stochastic resonance in a nonlinear model of a rotating, stratified shear flow, with a simple stochastic inertia-gravity wave parameterization
}

\author{
P. D. Williams ${ }^{1, *}$, T. W. N. Haine ${ }^{2}$, and P. L. $\operatorname{Read}^{1}$ \\ ${ }^{1}$ Atmospheric, Oceanic and Planetary Physics, Clarendon Laboratory, University of Oxford, UK \\ *Present address: Centre for Global Atmospheric Modelling, Department of Meteorology, University of Reading, UK \\ ${ }^{2}$ Department of Earth and Planetary Sciences, Johns Hopkins University, Baltimore, USA
}

Received: 17 September 2003 - Revised: 16 January 2004 - Accepted: 23 January 2004 - Published: 25 February 2004

\begin{abstract}
We report on a numerical study of the impact of short, fast inertia-gravity waves on the large-scale, slowlyevolving flow with which they co-exist. A nonlinear quasigeostrophic numerical model of a stratified shear flow is used to simulate, at reasonably high resolution, the evolution of a large-scale mode which grows due to baroclinic instability and equilibrates at finite amplitude. Ageostrophic inertiagravity modes are filtered out of the model by construction, but their effects on the balanced flow are incorporated using a simple stochastic parameterization of the potential vorticity anomalies which they induce. The model simulates a rotating, two-layer annulus laboratory experiment, in which we recently observed systematic inertia-gravity wave generation by an evolving, large-scale flow.
\end{abstract}

We find that the impact of the small-amplitude stochastic contribution to the potential vorticity tendency, on the model balanced flow, is generally small, as expected. In certain circumstances, however, the parameterized fast waves can exert a dominant influence. In a flow which is baroclinicallyunstable to a range of zonal wavenumbers, and in which there is a close match between the growth rates of the multiple modes, the stochastic waves can strongly affect wavenumber selection. This is illustrated by a flow in which the parameterized fast modes dramatically re-partition the probabilitydensity function for equilibrated large-scale zonal wavenumber. In a second case study, the stochastic perturbations are shown to force spontaneous wavenumber transitions in the large-scale flow, which do not occur in their absence. These phenomena are due to a stochastic resonance effect. They add to the evidence that deterministic parameterizations in general circulation models, of subgrid-scale processes such as gravity wave drag, cannot always adequately capture the full details of the nonlinear interaction.

Correspondence to: P. D. Williams

(williams@met.rdg.ac.uk)

\section{Introduction}

Inertia-gravity waves (IGWs) are ubiquitous throughout the stratified parts of the Earth's atmosphere and ocean. They are generated by the large-scale flow via three independent dynamical mechanisms: interactions with topography (e.g. Hines, 1988); spontaneous-adjustment radiation emitted as the large-scale flow adjusts (e.g. Ford et al., 2000); and as Kelvin-Helmholtz modes which develop due to local shear instabilities (e.g. Roach, 1970). Direct forcing of the atmosphere on the ocean mixed layer, and scattering of large-scale waves (e.g. the barotropic ocean tide) are two further generation mechanisms, which could be considered within the aforementioned categories but which are sufficiently important to merit an explicit mention. The characteristic length and time scales of IGWs are typically at least an order of magnitude smaller than those of the modes of main meteorological and climatological interest. This fact is frequently invoked to justify filtering IGWs from numerical weather prediction and climate models, under the assumption that interactions between motions on widely different scales are negligible. It is usual to attempt to account for the missing modes in the filtered model by including a parameterization of their expected effects. The full governing equations remain nonlinear, however. This means that a parameterized treatment of the fast modes cannot be rigorously justified, and leaves open the possibility of a significant nonlinear interaction between the IGW modes and the large-scale flow.

Most conventional parameterization schemes are deterministic, i.e. they describe the effects of sub-gridscale processes by a reproducible prescription which depends upon local resolved scale variables and a number of adjustable parameters (Palmer, 2001). For example, a well-known deterministic parameterization is that for momentum deposition due a continuous spectrum of stratospheric gravity waves, developed by Hines (1997). Recently, it has been shown that the deterministic Hines parameterization significantly underestimates the variability of the quasi-biennial oscillation, in simulations using the UK Meteorological Office Unified 
Model (C. Piani and W. Norton, private communication). A stochastic parameterization, in which an adjustable parameter is allowed to vary randomly according to some chosen probability distribution, gives an increased variability and better agreement with observations.

It is perhaps not surprising that stochastic representations of neglected processes can perform better than deterministic parameterizations, for the following reason. Filtering IGW motions from a numerical model leads to a corresponding reduction in the number of degrees of freedom of the system. This so-called balance assumption constrains the phase space trajectories to a reduced-dimensional subsurface of the full phase space, known as the slow manifold. Incorporating a deterministic parameterization of the neglected processes does not increase the number of degrees of freedom, because such parameterizations are closed, i.e. written in terms of model variables which already exist. The dynamics of the filtered system with and without the parameterization is therefore qualitatively similar. However, the random noise terms associated with a stochastic parameterization introduce new (non-deterministic) degrees of freedom, since each random number drawn from any probability-density function can be associated with a new and independent phase space co-ordinate, which is no less dynamically active than the coordinates associated with the deterministic degrees of freedom. Increasing the number of degrees of freedom this way suggests that stochastic parameterizations could give a better correspondence with the real system, because there is a closer match between the dimensionality of the phase spaces which they explore. In other words, the additional degrees of freedom introduced by stochastic terms in the filtered equations may be able to compensate, at least partially, for the degrees of freedom lost by the filtering.

This was essentially the finding of Palmer (2001), who filtered the least significant empirical orthogonal function (EOF) from the Lorenz (1963) equations, and considered both deterministic and stochastic parameterizations of the dynamical impacts of this neglected EOF. He found that the stochastic representation gave the best agreement, in terms of the mean state and internal variability, with the attractor of the original, unfiltered equations.

Until recently, research into the strength of the scaleseparated wave-wave interaction focused on greatly simplified numerical and theoretical models (e.g. Lorenz, 1986). Lovegrove et al. (2000) discovered that IGWs could be generated by an evolving large-scale flow in a rotating, two-layer annulus laboratory experiment, however. For the first time, the interaction could be studied in a real fluid, without the ad hoc approximations of highly-truncated and approximated models.

Most recently, we have explored these rotating annulus laboratory experiments exhibiting the co-existence of largescale and IGW modes. The spatio-temporal locations of IGWs are consistent with generation by the large-scale mode according to the spontaneous-adjustment radiation mechanism (Williams et al., 2003). We find that, when the system is devoid of IGWs, the large-scale flow exhibits a reluctance to undergo transitions between flows of different zonal wavenumber. In contrast, the flow undergoes such transitions much more readily when IGWs are present, even though typical IGW amplitudes are smaller than the balanced mode amplitude by a factor of around ten. The present study attempts to obtain independent corroborative evidence to support these laboratory findings, using a filtered numerical model. In particular, we are interested in determining whether a simple stochastic IGW parameterization is capable of reproducing the salient aspects of the laboratory results.

This paper is laid out as follows. In Sect. 2, we give brief details of the numerical model. IGWs are filtered from the model equations, and so we employ the model to simulate the large-scale modes only. We describe model runs both with and without stochastic forcing. In Sect. 3, we present two case study integrations which illustrate the impact of the stochastic forcing on wavenumber selection, and which demonstrate that the forcing can cause spontaneous transitions between different flow states. Finally, in Sect. 4, we discuss the results, and make a comparison with the corresponding laboratory findings. We consider the implications for flows in the atmosphere and ocean, as simulated by general circulation models, and finish with our conclusions.

\section{The QUAGMIRE numerical model}

For the numerical simulations in the present study, we employ a two-layer quasi-geostrophic annulus model known as QUAGMIRE (QUAsi-Geostrophic Model for Investigating Rotating fluids Experiments). A summary of the main model details is given here, and the reader is referred to Williams (2003) for a full technical description. Corresponding to the laboratory experiment (Williams et al., 2003), the model annulus has an inner vertical sidewall of radius $r_{1}=6.25 \mathrm{~cm}$, an outer vertical sidewall of radius $r_{2}=12.5 \mathrm{~cm}$, and a total depth of $2 H=25.0 \mathrm{~cm}$. The base and lid are both horizontal and flat. The base and sidewalls rotate with angular velocity $\Omega$ about the axis of symmetry, and the lid (in contact with the upper fluid layer) rotates relative to the base and sidewalls with angular velocity $\Delta \Omega$. This differential rotation provides a velocity shear which generates a large-scale mode due to baroclinic instability.

The model integrates the quasi-geostrophic potential vorticity equations in cylindrical co-ordinates in two stably-stratified incompressible layers, which have equal resting depths of $H=12.5 \mathrm{~cm}$. As in the laboratory experiment, the upper and lower layer densities and kinematic viscosities are $\rho_{\text {upper }}=997 \mathrm{~kg} \mathrm{~m}^{-3}$, $\rho_{\text {lower }}=1003 \mathrm{~kg} \mathrm{~m}^{-3}, \quad \nu_{\text {upper }}=1.27 \times 10^{-6} \mathrm{~m}^{2} \mathrm{~s}^{-1}$ and $v_{\text {lower }}=1.08 \times 10^{-6} \mathrm{~m}^{2} \mathrm{~s}^{-1}$. The mutual interfacial tension is $T=2.85 \times 10^{-2} \mathrm{Nm}^{-1}$. The fluids are forced by Ekman pumping and suction velocities at the lid, base and interface, and the effects of weak interfacial tension are included. We use a regular model grid of 96 points in azimuth and 16 points in radius. We timestep the potential vorticity tendency equations in physical space, but transform to vertical and 
azimuthal normal mode space once per timestep to obtain the streamfunction by inverting the potential vorticity. The timestep, chosen to give a Courant number of 0.01 , is typically $0.01 \mathrm{~s}$.

We use the Arakawa (1966) second order Jacobian for the advection terms; a leap-frog scheme with a Robert (1966) three-level time filter to suppress computational mode-splitting; Ekman layer diffusion terms time-lagged by one step for stability; and a second order horizontal potential vorticity hyperdiffusion to suppress gridscale energy buildup. At the lateral boundaries, we apply impermeability to the eddy components, and impose no-slip boundary conditions on the axisymmetric component which develops as a correction to the mean flow. Multiple test integrations were performed to demonstrate insensitivity of the model output to the numerical hyperdiffusivity, Robert filter parameter, gridspacing and timestep.

We next describe, in turn, sample model runs both without, and then with, a stochastic forcing term added to the governing potential vorticity equations.

\subsection{Model runs without stochastic forcing}

There is an equilibrium solution to the deterministic model equations, corresponding to solid-body rotation in both layers, at different rates (e.g. Hart, 1973). We assess the stability of this state by using spun-up initial conditions, given by the equilibrium flow seeded with superimposed small-amplitude random noise. The noise provides a small perturbation, containing energy at all resolveable wavenumbers, from which any unstable quasi-geostrophic mode can grow. The development of a large-scale wave due to baroclinic instability, during a typical model run which had $\Omega=3.50 \mathrm{rad} \mathrm{s}^{-1}$ and $\Delta \Omega=0.08 \mathrm{rad} \mathrm{s}^{-1}$, is shown in Fig. 1. Values of the Rossby number, Ro, internal Froude number, Fr, and dissipation parameter, $d$, defined by

Ro $=\frac{\Delta \Omega}{2 \Omega}$,

$\mathrm{Fr}=\frac{4 \Omega^{2}\left(r_{2}-r_{1}\right)^{2}}{g^{\prime} H}$

and

$d=\frac{\sqrt{\bar{\nu} \Omega}}{H \Delta \Omega}$,

are given in the figure caption. In these equations, $g^{\prime} \equiv 2 g\left(\rho_{\text {lower }}-\rho_{\text {upper }}\right) /\left(\rho_{\text {lower }}+\rho_{\text {upper }}\right)=6 \mathrm{~cm} \mathrm{~s}^{-2}$ is the reduced gravity and $\bar{v} \equiv\left(\nu_{\text {upper }}+v_{\text {lower }}\right) / 2=1.18$ $\times 10^{-6} \mathrm{~m}^{2} \mathrm{~s}^{-1}$ is the mean kinematic viscosity. There is an initial transient period during which short-lived radial and zonal modes emerge from the noise. Then, a particular single mode grows to dominate the flow and equilibrates, drifting slowly around the annulus at a constant, finite amplitude. Though the model can simulate flows in which the equilibrated amplitude vacillates, i.e. periodically grows and decays with time, such flows are not studied in the present work. The final equilibrated mode (here of zonal wavenumber 3 ) is not necessarily that with the largest initial growth rate (here of zonal wavenumber 5), in concordance with the analytical investigation of Appleby (1988).

As in the laboratory experiments, the zonal wavenumber, phase speed and amplitude of the equilibrated wave depend upon $\Omega$ and $\Delta \Omega$. Examples of typical equilibrated wavenumber 1 and 2 flows are shown in Fig. 2. Hundreds of model runs have been performed, each with slightly different combinations of $\Omega$ and $\Delta \Omega$ ranging from $\Omega=1.0 \mathrm{rads}^{-1}$ to $\Omega=3.5 \mathrm{rad} \mathrm{s}^{-1}$, and from $\Delta \Omega=0.01 \mathrm{rad} \mathrm{s}^{-1}$ to $\Delta \Omega=1.6 \mathrm{rad} \mathrm{s}^{-1}$. In each run, the perturbations in the initial state are found to either decay (baroclinic stability), or to grow to a mode with zonal wavenumber 1, 2 or 3 (baroclinic instability) as illustrated in Figs. 2(a), 2(b) and 1(f), respectively. When model experiments with the same $[\Omega, \Delta \Omega]$ are repeated many times with different noise fields in the initial states, the same equilibrated wavenumber is usually found. This implies that the equilibrated flow is insensitive to the precise details of the initial state. The exception to this insensitivity is at very large rotation rates $\left(\Omega>3 \mathrm{rad} \mathrm{s}^{-1}\right.$ and $\left.\Delta \Omega>1 \mathrm{rad} \mathrm{s}^{-1}\right)$, where reproducibility of the post-transient state is not always observed. This high-rotation regime is not examined in the present study.

There is reasonable agreement between the laboratory and model azimuthal wavenumber regime diagrams in the twodimensional space defined by $[\Omega, \Delta \Omega]$ (Williams, 2003). This fact indicates that the impact of the IGWs on the largescale flow is generally small, because the laboratory flow contains ubiquitous IGWs but the model contains none. So, in the present case, a filtered model seems capable of adequately simulating flows in which unresolved motions occur. This is a signal of a negligible interaction. Nevertheless, we will present examples in Sect. 3 of special cases in which the parameterized IGWs exert a strong influence on the global flow.

\subsection{Model runs with stochastic forcing}

We now include a simple stochastic parameterization of IGWs in QUAGMIRE, to mimic the effects of the IGWs in the laboratory experiments. We do this by adding a random noise term to the right side of the prognostic model equations for each layer. An implicit assumption is that the precise details and structure of the laboratory small-scale waves are irrelevant, and that they have the same impact on the balanced flow as would random fluctuations.

The quasi-geostrophic model QUAGMIRE cannot capture the evolution of the IGWs, which are inherently ageostrophic. However, we can reasonably expect it to capture the response of the quasi-geostrophic modes to potential vorticity anomalies induced by the small-scale modes. IGWs have zero potential vorticity anomaly only in the linear limit, but we assert that finite amplitude IGWs may carry a non-zero perturbation potential vorticity (PPV). It is this quantity which we parameterize in the model equations, as a stochastic perturbation to the PPV tendency fields. Note 

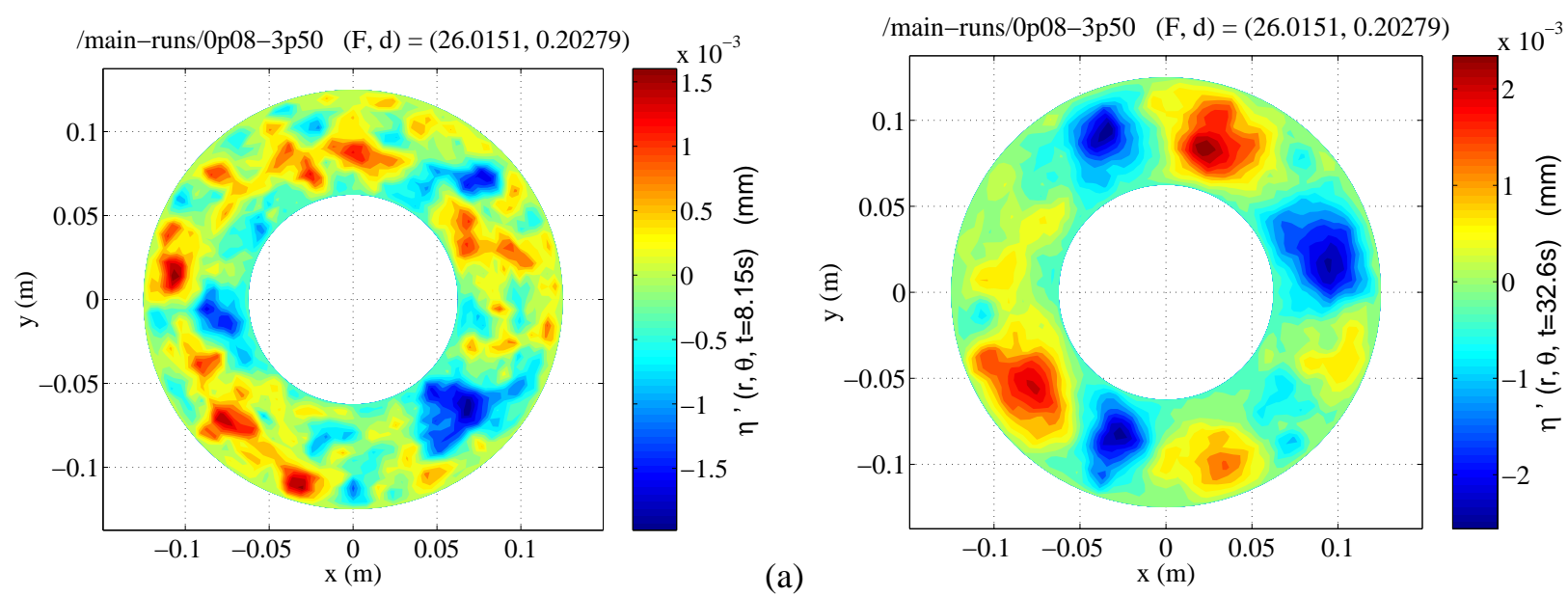

(b)
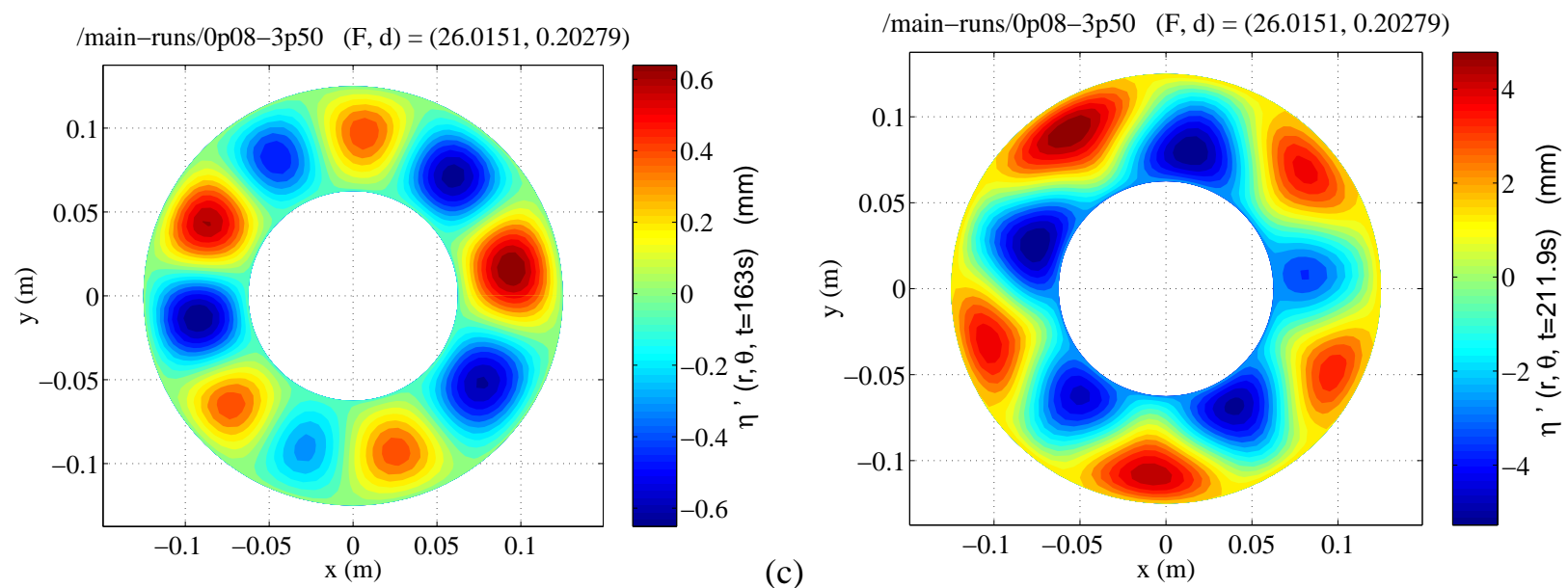

(d)
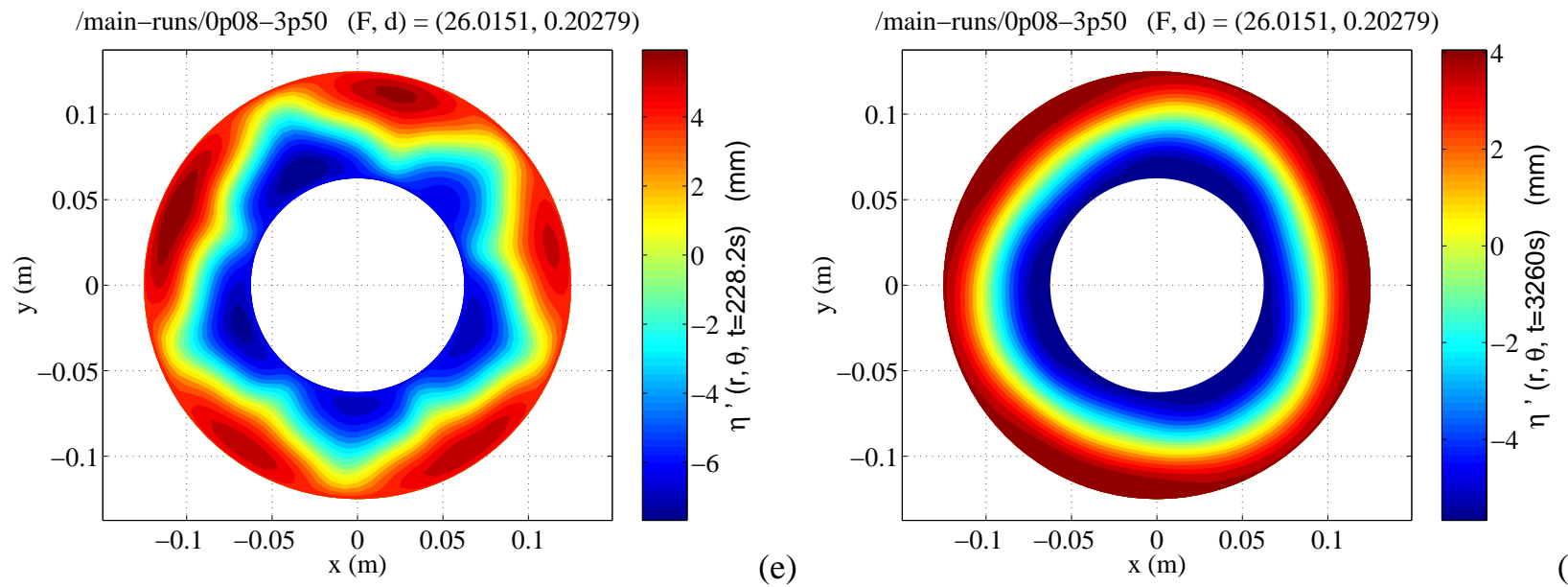

(f)

Fig. 1. Plots of perturbations to the equilibrium lower layer depth field, for the case $\Omega=3.50 \mathrm{rad} \mathrm{s}^{-1}$ and $\Delta \Omega=0.08 \mathrm{rad} \mathrm{s}^{-1}$, at which $\mathrm{Ro}=0.011, \mathrm{Fr}=26$ and $d=0.20$. The plots are ordered in time (right label), and show the evolution of the model state from random smallamplitude initial conditions in (a), to an equilibrated large-scale mode with azimuthal wavenumber 3 in (f). Note that the colourbar scales vary between the plots. 


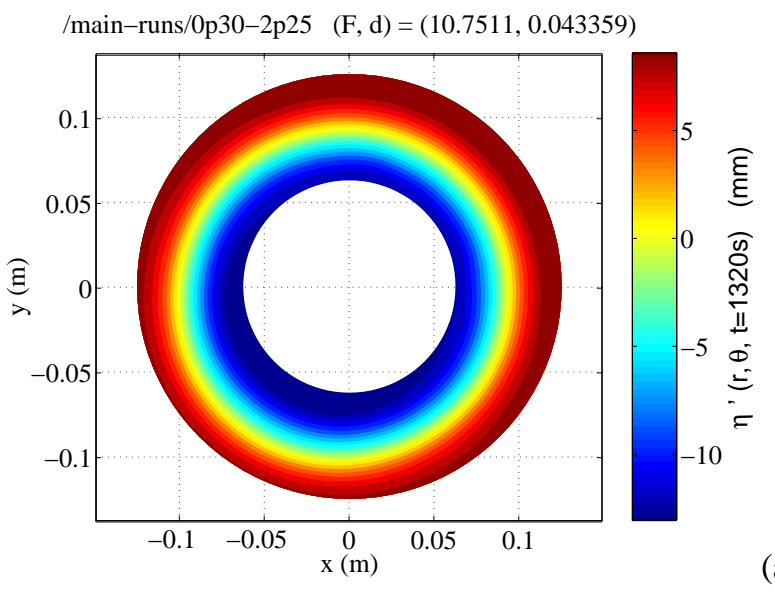

(a)

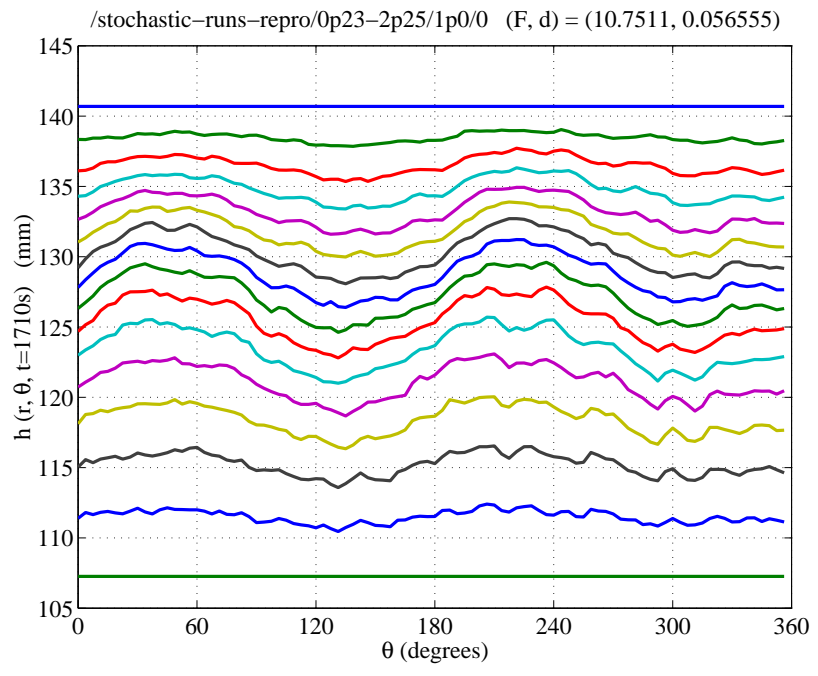

Fig. 3. Zonal profiles of lower layer depth, long after equilibration in the presence of stochastic forcing of amplitude $a=1.0 \mathrm{~s}^{-2}$. The rotation rates are $\Omega=2.25 \mathrm{rad} \mathrm{s}^{-1}$ and $\Delta \Omega=0.23 \mathrm{rad} \mathrm{s}^{-1}$, at which $\operatorname{Ro}=0.051, \mathrm{Fr}=11$ and $d=0.057$. Profiles are shown at each of the 16 radii at which there are model gridpoints, ranging from $62.5 \mathrm{~mm}$ (top, blue curve) to $125.0 \mathrm{~mm}$ (bottom, green curve). At the inner and outer sidewall boundaries the lower layer depth displays no variation with azimuth, because impermeable boundary conditions are imposed there. These are profiles of total lower layer depth, as opposed to the plots in Figs. 1 and 2, which show perturbations to the equilibrium solution discussed in Sect. 2.1.

The primary objective of our chosen parameterization scheme is simplicity. The noisy stochastic forcing fields which we use take the simplest conceivable form, namely additive contributions drawn from a uniform distribution of constant width, with no spatial or temporal auto-correlations. The parameterization could clearly be refined, for example by allowing the width of the probability density function to vary with the underlying potential vorticity gradient, or by including spatio-temporal auto-correlations which satisfy the internal inertia-gravity wave dispersion relation. It seems highly unlikely that such refinements would alter the qualitative conclusions which we are able to reach with the simple scheme, however. In support of this assertion, the qualitative aspects of the quasi-biennial oscillation and annual cycle in a model with stochastic inertia-gravity wave drag, are insensitive to whether the Hines parameter is drawn from a normal or exponential distribution (C. Piani and W. Norton, private communication).

Apart from some important exceptions, to be discussed in Sect. 3, the impact of the stochastic terms on the equilibrated large-scale flow is generally small. Across most of the $[\Omega, \Delta \Omega]$ regime diagram, the post-transient wavenumber and wave speed are not detectably modified by the new terms. There is a slight increase in large-scale wave amplitude, as energy from the stochastic small-scale waves filters up-scale. Typical zonal profiles of lower layer depth in the 


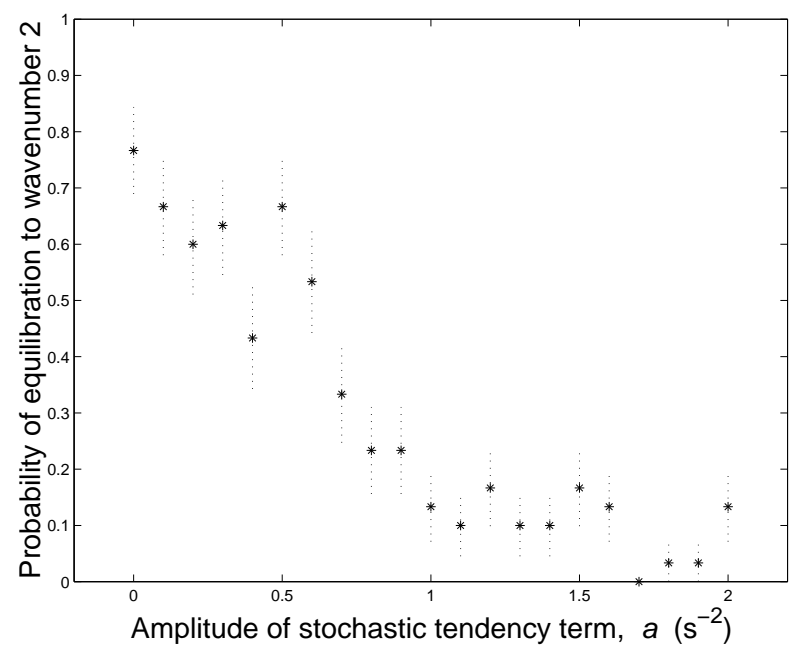

Fig. 4. Probability of equilibration to azimuthal wavenumber two as a function of stochastic noise amplitude, $a$. The rotation rates are $\Delta \Omega=0.23 \mathrm{rad} \mathrm{s}^{-1}$ and $\Omega=2.25 \mathrm{rad} \mathrm{s}^{-1}$, at which $\mathrm{Ro}=0.051$, $\mathrm{Fr}=11$ and $d=0.057$. For each probability, $p$, the error bars are obtained from the standard deviation $\sqrt{N p(1-p)}$ of the binomial distribution $\mathrm{B}(N, p)$, with $N=30$.

presence of the stochastic forcing are shown in Fig. 3, in which the zonal wavenumber of the large-scale mode is 2 . The effects of the stochastic parameterization on the flow are clear, as a small-amplitude gridscale perturbation to the main wavenumber 2 signal, which is much smoother when the parameterization is not activated. The interface amplitude of the balanced mode reaches a maximum of around $3 \mathrm{~mm}$ near mid-radius, whilst the characteristic anomaly associated with the stochastic terms is seen to be around a factor of 10 smaller. These typical model amplitudes are similar to those of the large- and small-scale waves as measured in the laboratory experiment (Williams, 2003).

\section{Results}

In this section, we use case studies of two particular model runs to illustrate the large impact that the stochasticallyparameterized fast waves can have on wavenumber selection and spontaneous transitions, at certain points in parameter space.

\subsection{Impacts on wavenumber selection}

We use a stochastically-forced numerical experiment with $\Delta \Omega=0.23 \mathrm{rad} \mathrm{s}^{-1}$ and $\Omega=2.25 \mathrm{rad} \mathrm{s}^{-1}$. This is quite close to the azimuthal wavenumber $1 \leftrightarrow 2$ transition curve in the $[\Omega, \Delta \Omega]$ regime diagram, which means that the linear growth rates of the wavenumber 1 and 2 modes are approximately equal. An ensemble of thirty members was carried out for each of various stochastic forcing amplitudes, ranging from $a=0$ to $a=2 \mathrm{~s}^{-2}$. Within each ensemble, the only difference between the thirty members was the particular random numbers in the stochastic forcing fields. As in Sect. 2, the initial conditions for the integrations were given by the equilibrium flow with superimposed small-amplitude noise, though it seems that the noise seeding is not necessary in this case as perturbations from which instabilities can grow are provided by the stochastic forcing. In each case, the equilibrated azimuthal wavenumber $m$ was noted, and found to be either 1 or 2

For each ensemble of constant noise amplitude, the probability of equilibration to wavenumber 2 was calculated and is plotted in Fig. 4. There is a clear and strong dependence of probability partition on noise amplitude. The results are consistent with a linear drop-off in the probability of $m=2$ as the noise increases to around $a=1.0 \mathrm{~s}^{-2}$, followed by a saturation at a probability of around $10 \%$ up to a noise of $a=2.0 \mathrm{~s}^{-2}$. At each noise amplitude, zonal profiles of lower layer depth (such as those in Fig. 3) still showed smooth, large-scale modes superimposed with small-amplitude, gridscale noise.

The addition of small-amplitude noise has had a very significant impact upon the predictability of the system's phase space attractor, at this point in parameter space. Ten-member ensembles at the centres of the $m=1,2,3$ regions of the regime diagram always showed equilibration to the given wavenumber, irrespective of noise amplitudes up to $2.0 \mathrm{~s}^{-2}$. This suggests that the regions of $[\Omega, \Delta \Omega]$ parameter space in which IGWs can exert a strong influence on large-scale mode wavenumber selection, are confined to finite width strips adjacent to transition curves, where the system is highly intransitive.

\subsection{Spontaneous transitions}

In Sect. 3.1, we investigated the stability of an equilibrium axisymmetric shear flow continuously seeded with stochastic noise, which is a simple model of an axisymmetric atmospheric jetstream in the presence of IGWs. A more likely scenario in the atmosphere is for a large-scale azimuthal mode to have already grown due to baroclinic instability, and reached a quasi-equilibrium at finite amplitude, giving a perturbation to the jetstream. There are therefore good geophysical reasons to be more interested in the stability of a finiteamplitude large-scale wave in the presence of IGWs, rather than the stability of an axisymmetric flow.

In order to investigate this, we now take a wavenumber 2 model flow with $\Delta \Omega=0.23 \mathrm{rad} \mathrm{s}^{-1}$ and $\Omega=2.25 \mathrm{rad} \mathrm{s}^{-1}$, which has equilibrated at finite amplitude in the absence of stochastic forcing. As in Sect. 3.1 (with these parameters), the system is quite close to the wavenumber $1 \leftrightarrow 2$ transition curve. In the present investigation, we continue the model integrations using the finite amplitude wavenumber 2 mode as an initial condition, but increase the stochastic noise amplitude from $a=0$ to $a=2.0 \mathrm{~s}^{-2}$, by $10^{-6} \mathrm{~s}^{-2}$ each timestep so that the increase is quasi-continuous.

Results show that the wavenumber 2 mode persists until the noise reaches a certain threshold level, at which point a 


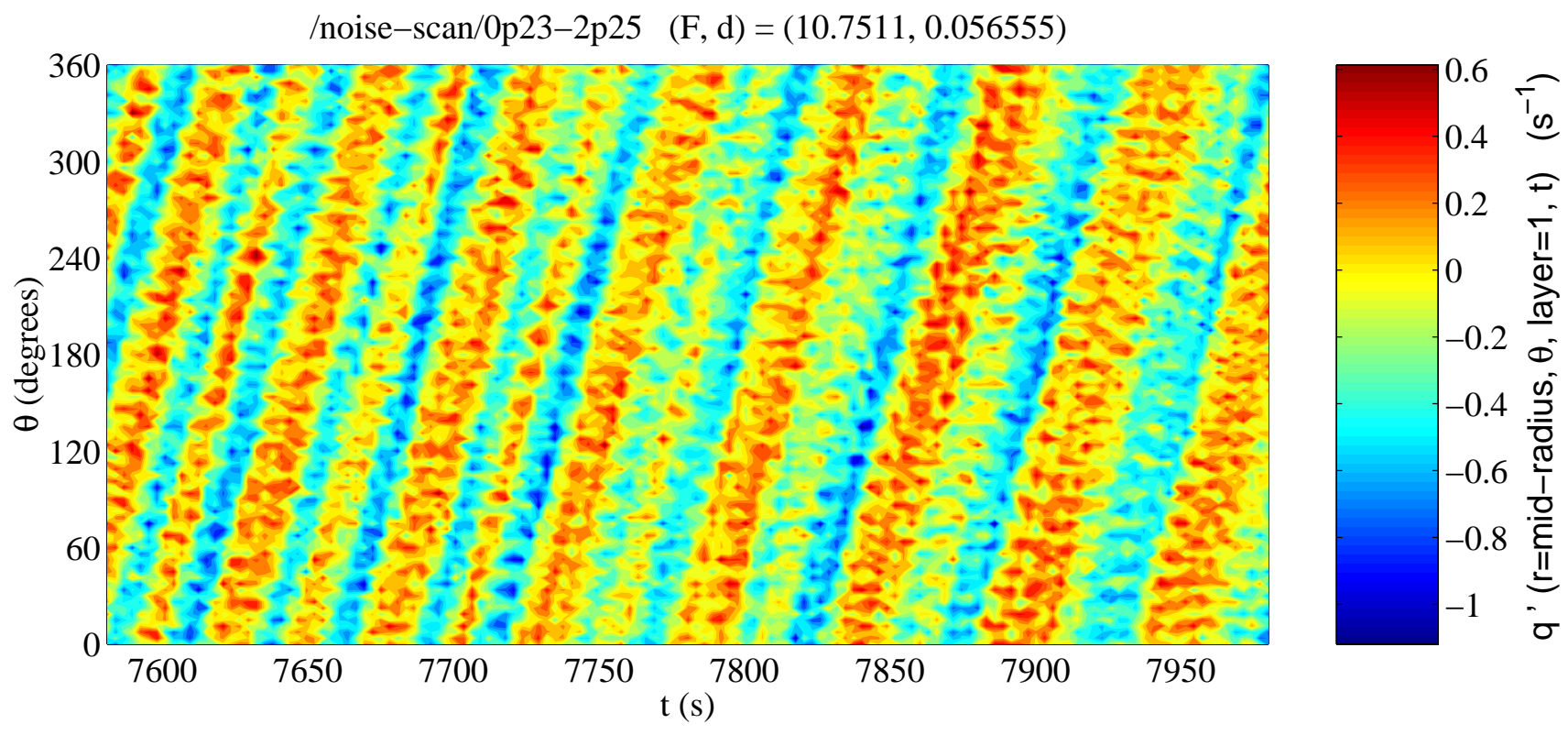

Fig. 5. Hovmüller diagram, showing an azimuth-time contour plot of model perturbation potential vorticity, at mid-radius $r=9.4 \mathrm{~cm}$ in the upper layer, at the time of a spontaneous wavenumber transition. The run parameters were $\Delta \Omega=0.23 \mathrm{rad} \mathrm{s}^{-1}$ and $\Omega=2.25 \mathrm{rad} \mathrm{s}^{-1}$, at which $\mathrm{Ro}=0.051, \mathrm{Fr}=11$ and $d=0.057$.

spontaneous transition is observed to a wavenumber 1 mode. A Hovmüller diagram showing the transition, which takes place over around $100 \mathrm{~s}$, which is the time taken for the largescale wave to travel around the annulus twice, is shown in Fig. 5. This kind of transition is never observed without the stochastic IGW parameterization activated, and so we conclude that the transition was caused by the parameterization. At the time of the transition, the stochastic noise parameter had reached a value of $a=1.1 \mathrm{~s}^{-2}$. By examining zonal profiles of lower layer depth, such as those in Fig. 3, at the transition time, we find that this corresponds to a gridscale interface anomaly of root-mean-square amplitude $0.3 \mathrm{~mm}$, which is much smaller than the amplitude of the large-scale mode.

At first sight, the perturbation potential vorticity plot in Fig. 5 may seem unrealistically noisy, but this is simply because the Laplacian operator, which is required to obtain the perturbation potential vorticity from the interface height, amplifies small scales relative to large scales. For this reason, the profiles of lower layer depth in Fig. 3 appear much less noisy.

After the transition to the wavenumber 1 mode, the stochastic noise amplitude $a$ was decreased back to zero by $10^{-6} \mathrm{~s}^{-2}$ each timestep, but the reverse transition back to wavenumber 2 did not occur. At the end of the integration, when the noise had reached zero, the wavenumber 1 mode was still dominant, indicating the presence of hysteresis in the system.

As in Sect. 3.1, when the above experiment was repeated with rotation rates corresponding to the centre of a wavenumber regime in parameter space, spontaneous transitions were not observed.

\section{Conclusions}

We have investigated the effects of including a simple stochastic inertia-gravity wave parameterization, in a quasigeostrophic model of a rotating, stratified shear flow. In general, the effect of the parameterized short waves on the large-scale, main modes is too small to be detected. The short waves therefore behave as linear superimposed features which do not seem to interact in an observable way with the large-scale flow. Sufficiently close to a wavenumber transition curve, however, a nonlinear effect allows the short waves to exert a strong influence over long wavelength mode selection. In particular, case studies have been given in Sect. 3 of model integrations in which the probability-density function for equilibrated wavenumber is substantially modified, and in which spontaneous transitions are observed between different azimuthal modes. Both of these effects are directly attributable to the presence of the stochastic short waves, and occur even though the characteristic high wavenumber amplitudes are at least an order of magnitude smaller than the long wave amplitude. It seems highly unlikely that these phenomena could be reproduced using a deterministic parameterization of the inertia-gravity waves, such as that developed by Hines (1988). Such a parameterization would merely lead to an additional small drag force on the fluids, adding slightly to the drag force due to the viscous boundary layers at the sidewalls, lid, base and interface.

We have seen that the addition of small-amplitude noise has had a very significant impact on the system dynamics. This phenomenon is a form of stochastic resonance (Pikovsky et al., 2001), since a small (stochastic) forcing produces a large (resonant) response. This is a nonlinear 


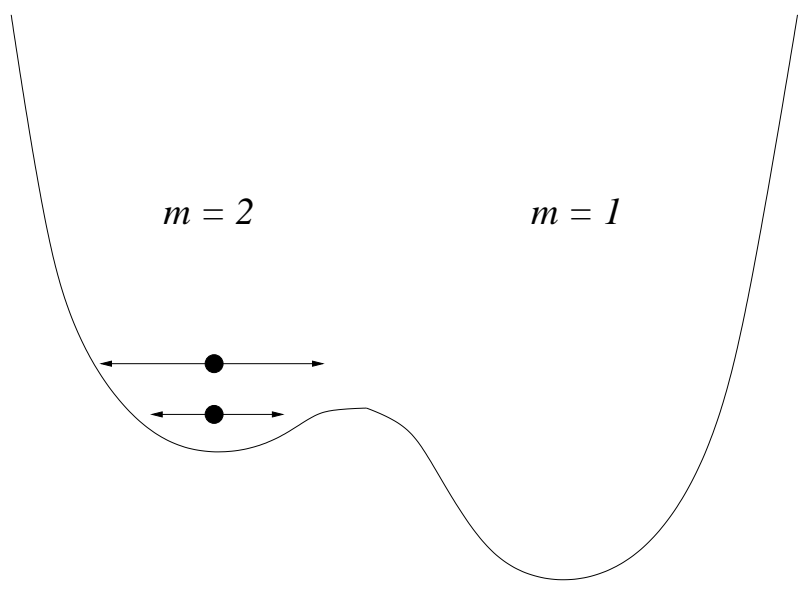

Fig. 6. Schematic double-well potential for a bistable system, which can explain the observed model regime transitions close to the $1 \leftrightarrow 2$ transition curve in $[\Omega, \Delta \Omega]$ parameter space.

resonance which is not dependent upon any matching of timescales, unlike the familiar criterion required for linear resonance. If stochastic resonance is exhibited by a nonlinear system, then the introduction of very small amplitude noise can dramatically affect the system state. For example, De Swart and Grasman (1987) have studied the effects of adding a stochastic forcing term to a low-order atmospheric spectral model based on the barotropic potential vorticity equation, and found that the noise forces the system to visit alternately different quasi-stable regimes.

The results of Sect. 3.2 suggest a simple schematic model for explaining the observed spontaneous transitions. We represent the stable equilibrium states $m=1$ and $m=2$ by minima of the potential well shown in Fig. 6. With the system in the $m=2$ state, a short burst of sufficiently strong stochastic forcing permits the system to overcome the transition barrier and thereby undergo an irreversible transition to the $m=1$ state, in which the system remains after the burst. Presumably, the barrier height increases with distance from a transition curve in the $[\Omega, \Delta \Omega]$ regime diagram, increasing the noise amplitude which is required to force a transition. This is consistent with the observation of Sect. 3.2 that, far away from regime diagram transition curves, even very large stochastic forcing is unable to induce a spontaneous transition.

If the stochastic forcing were sufficiently large, it could begin to dominate the model output and give flows with wavenumbers which were essentially random. In that case, both of the wavenumber regimes in the experiments of Sect. 3.1 might be expected to have an equal probability of occurrence. The stochastic forcing which we have used is much smaller than this, however. It can be large enough to push the system state over the transition barrier in Fig. 6, in the direction from $m=2$ to $m=1$, but is never large enough to effect the reverse transition. This is consistent with the change in wavenumber two probability from 0.8 to 0.1 as the noise is increased, as shown in Fig. 4. Presumably if the noise were increased further still, a point would be reached at which the noise began to dominate the signal and, correspondingly, the probability might be expected to tend to 0.5 . We have not investigated this regime, as its geophysical relevance is extremely limited.

The present findings are bourne out by laboratory observations. We have found in the numerical study that the presence of stochastically-parameterized inertia-gravity waves increases the likelihood of a model state transition. Correspondingly, we have found in the laboratory annulus experiments that the flow without inertia-gravity waves exhibits a reluctance to undergo transitions which take place readily in their presence. The appearance of inertia-gravity waves in the laboratory is related to intrinsic fluid properties, whose effects are scale-selective and so suppress or permit inertiagravity wave activity without modifying the large-scale mode (Williams, 2003). For example, we have been able to modify the interfacial tension using a chemical surfactant, and thereby completely suppress inertia-gravity waves.

Since laboratory inertia-gravity wave amplitudes cannot be continuously increased in a controlled way, in contrast with the numerical experiments, we instead choose to continuously increase the background rotation rate. As an example, laboratory experiments were run in which $\Omega$ was gradually increased at a rate of $4 \times 10^{-4} \mathrm{rad} \mathrm{s}^{-2}$, starting from $2.3 \mathrm{rad} \mathrm{s}^{-1}$ at which the equilibrated flow had azimuthal wavenumber 2. $\Delta \Omega$ was held constant at $0.62 \mathrm{rad} \mathrm{s}^{-1}$. Without inertia-gravity waves, the flow made a transition to a wavenumber 3 state when $\Omega$ had reached $3.4 \mathrm{rad} \mathrm{s}^{-1}$, but with inertia-gravity waves, the transition occurred earlier, when $\Omega$ reached $2.7 \mathrm{rad} \mathrm{s}^{-1}$. The values of the nondimensional parameters for the transition were $\mathrm{Ro}=0.09, \mathrm{Fr}=25$ and $d=0.026$ in the absence of inertia-gravity waves, and $\mathrm{Ro}=0.11, \mathrm{Fr}=16$ and $d=0.023$ in their presence. Inertiagravity waves therefore seem to have a strong influence on transitions, both in the laboratory and the model. There are laboratory regimes in which the wavenumber 1 and 2 states vacillate irregularly, rather than a single state remaining steady. This has a closer correspondence with the atmospheric regime, and so an investigation of inertia-gravity wave impacts on these states should form an important component of any future work.

The Rossby, Froude and dissipation numbers for the laboratory experiment, atmosphere and ocean are not dissimilar (Williams, 2003), though the non-dimensionalized viscosity and interfacial tension are much larger in the laboratory. It therefore seems likely that the qualitative findings of this study apply to the atmosphere and ocean. The implication is that a short, intense burst of inertia-gravity waves could force a large-scale regime change which persists long after the inertia-gravity waves have dissipated. Such an event is unlikely to be captured by a deterministic inertia-gravity wave parameterization, as currently used by most operational meteorological centres. There is a clear need for further research to investigate the likely forecast error that this phenomenon could inflict. Moreover, stochastic inertia-gravity wave parameterizations would seem to offer a promising and convenient way of capturing it. 
Acknowledgements. PDW acknowledges support under a research studentship from the UK Natural Environment Research Council, held at the University of Oxford, with award reference number GT04/1999/AS/0203.

Edited by: R. Grimshaw

Reviewed by: T. N. Palmer and another referee

\section{References}

Appleby, J. C.: Selection of baroclinic waves, Quart. J. Roy. Meteorol. Soc., 114, 1173-1179, 1988.

Arakawa, A.: Computational design for long-term numerical integration of the equations of fluid motion: two-dimensional incompressible flow, J. Comp. Phys., 1, 119-143, 1966.

De Swart, H. E. and Grasman, J.: Effect of stochastic perturbations on a low-order spectral model of the atmospheric circulation, Tellus, 39A, 10-24, 1987.

Ford, R., McIntyre, M. E., and Norton, W. A.: Balance and the slow quasimanifold: some explicit results, J. Atmos. Sci., 57, 12361254, 2000

Hart, J. E.: On the behaviour of large-amplitude baroclinic waves, J. Atmos. Sci., 30, 1017-1034, 1973.

Hines, C. O.: Tropopausal mountain waves over Arecibo: a case study, J. Atmos. Sci., 46, 476-488, 1988.

Hines, C. O.: Doppler spread parameterization of gravity wave momentum deposition in the middle atmosphere, Part 1: Basic formulation; Part 2: Broad and quasi monochromatic spectra and implementation, J. Atmos. Solar Terr. Phys., 59, 371-400, 1997.
Lorenz, E. N.: Deterministic nonperiodic flow, J. Atmos. Sci., 42, 433-471, 1963.

Lorenz, E. N.: On the existence of a slow manifold, J. Atmos. Sci., 43, 1547-1557, 1986.

Lovegrove, A. F., Read, P. L., and Richards, C. J.: Generation of inertia-gravity waves in a baroclinically unstable fluid, Quart. J. Roy. Meteorol. Soc., 126, 3233-3254, 2000.

Palmer, T. N.: A nonlinear dynamical perspective on model error: a proposal for non-local stochastic-dynamic parameterization in weather and climate prediction models, Quart. J. Roy. Meteorol. Soc., 127, 279-304, 2001.

Pikovsky, A., Rosenblum, M., and Kurths, J.: Synchronization: a universal concept in nonlinear sciences, Cambridge University Press, 2001.

Roach, W. T.: On the influence of synoptic development on the production of high level turbulence, Quart. J. Roy. Meteorol. Soc., 96, 413-429, 1970.

Robert, A. J.: The integration of a low order spectral form of the primitive meteorological equations, J. Meteor. Soc. Japan, 44(5), 237-245, 1966.

Williams, P. D.: Nonlinear interactions of fast and slow modes in rotating, stratified fluid flows, Ph.D. thesis, Oxford University, 2003.

Williams, P. D., Read, P. L., and Haine, T. W. N.: Spontaneous generation and impact of inertia-gravity waves in a stratified, two-layer shear flow, Geophys. Res. Lett., 30, 2255, doi:10.1029/2003GL018498, 2003. 